EOMmUn: Communication et organisation

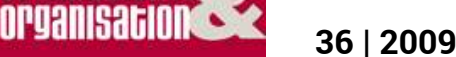

Pour une approche communicationnelle de l'individu au travail

\title{
Reconnaissance et communication : une logique de l'assujettissement
}

\section{Thomas Heller}

\section{OpenEdition}

\section{Journals}

Édition électronique

URL : http://journals.openedition.org/communicationorganisation/950

DOI : 10.4000/communicationorganisation.950

ISSN : 1775-3546

Éditeur

Presses universitaires de Bordeaux

Édition imprimée

Date de publication : 1 décembre 2009

Pagination : 108-120

ISBN : 978-2-86781-719-9

ISSN : $1168-5549$

Référence électronique

Thomas Heller, « Reconnaissance et communication : une logique de l'assujettissement »,

Communication et organisation [En ligne], 36|2009, mis en ligne le 14 mars 2011, consulté le 19 avril

2019. URL : http://journals.openedition.org/communicationorganisation/950 ; DOI : 10.4000/

communicationorganisation.950 
Dossier : Pour une approche communicationnelle de l'individu au travail

\begin{abstract}
Résumé
Dans un article intitulé Le sujet et le pouvoir, le philosophe Michel Foucault évoque une forme de pouvoir qui lie l'individu à lui-même et assure ainsi sa soumission aux autres. Cette formule qui exprime les deux versants de l'assujettissement est le point de départ d'une analyse des implications assujettissantes de pratiques et situations de communication en entreprise. Le concept de reconnaissance dans sa double acception d'identification et d'affirmation, permet de mettre en évidence comment opère la logique assujettissante de la communication, qui caractérise une dimension particulière de la gouvernementalité des salariés.
\end{abstract}

\title{
Mots-clefs
}

Reconnaissance, assujettissement, gouvernement, identité, soi, soumission.

\begin{abstract}
In a paper entitled subject and power, the philosopher Michel Foucault talks about a power which links man to himself, to his identity, and ensures in this way his submission to the others. This expression which talks about subjection/constraint is the starting point of an analysis about the governmental and subjecting implications of practices and situations of communication in firms. The concept of recognition, as identification and affirmation, allows to bring to the fore how this subjecting logic of communication which characterizes a part of actual employees governmentality operates.
\end{abstract}

\section{Keywords}

Recognition, subjection, government, identity, self, submission

Thomas HELLE $\mathbf{R}$ est maître de conférences en Sciences de l'Information et de la Communication à l'Université de Lille 1. Il est chercheur au GERIICO (Université de Lille 3), au sein de l'équipe COPI (Communications organisationnelles). Ses travaux portent sur les rapports de la communication au gouvernement des salariés, sur les enjeux identitaires et politiques de la communication en entreprise. Ils portent également sur les représentations filmiques du monde salarial et leurs enjeux idéologiques et sociopolitiques. 


\section{Reconnaissance et communication : une logique de l'assujettissement Thomas Heller}

thomas.heller@univ-lille1.fr

Dans un article intitulé le sujet et le pouvoir, M. Foucault propose une typologie des luttes en distinguant trois sortes d'enjeu : lutte contre l'exploitation, lutte contre les dominations (ethnique, sociale, religieuse) et enfin lutte contre «tout ce qui lie l'individu à lui-même et assure ainsi sa soumission aux autres » (1994a : 227). À travers ces différentes luttes, c'est la question du pouvoir, de son exercice, de son lieu d'application et de ses effets qui est posée. Ce qui nous intéresse ici c'est cette idée d'un processus qui noue ensemble constitution de soi et soumission. L'attention accordée aujourd'hui dans de nombreuses entreprises à la singularité, à la subjectivité, à l'identité du salarié ${ }^{44}$, portée par les discours managériaux et repérables dans certaines pratiques de gestion (évaluation du «savoir-être », développement personnel, etc.) invite effectivement à rapporter ces pratiques et discours à cette idée, en raison même du rapport soi/gestion qu'ils impliquent. Mais comment penser un tel pouvoir, comment expliquer ce lien entre constitution de soi et soumission à l'autre, tel que le second découle du premier? L'hypothèse que nous défendons ici est qu'un tel pouvoir caractérise un enjeu de certaines formes de communication au sein des entreprises. Saisir cet enjeu, c'est donc mettre au jour comment un tel lien opère dans la communication ; il nous semble que le concept de reconnaissance, en regard de ses différentes acceptions, permet de comprendre cette opération. Autrement dit, la reconnaissance est envisagée comme une entrée pertinente pour décrire la communication dans les termes d'un assujettissement.

\section{Du gouvernement des salariés à la communication en entreprise}

Une particularité de la notion d'assujettissement chez Foucault, et ce qui en fait tout son intérêt, c'est qu'elle désigne à la fois une

\footnotetext{
44 Le développement, ces dernières décennies, de formes variées d'organisation du travail, d'encadrements juridiques des relations employeurs employés et des rémunérations rend plus problématique l'usage de la notion de salarié. Elle garde néanmoins toute sa pertinence si l'on retient de celle-ci qu'elle renvoie à une place dans les rapports de production caractérisée par la dépendance et la subordination.
} 
Dossier : Pour une approche communicationnelle de l'individu au travail

soumission à l'autre "par le contrôle et la dépendance", et la formation d'un rapport à soi, de l'attachement «à sa propre identité par la conscience ou la connaissance de soi » (1994a : 227), constitué par un pouvoir. Foucault parle aussi de «soumission de la subjectivité » (1994a : 228) pour caractériser ce rapport à soi comme assujettissement. L'articulation entre ces deux formes d'assujettissement renvoie à la notion de gouvernementalité qui, dans les derniers écrits du philosophe, désigne «la rencontre entre les techniques de domination exercée sur les autres et les techniques de soi $\gg(1994 b$ : 785).

Le gouvernement des individus dans les entreprises, qui consiste à «structurer le champ d'actions éventuelles des autres» (Foucault, 1994a : 237), est une nécessité qui découle de trois caractéristiques du procès de production: d'une part, la possibilité d'atteindre collectivement des objectifs impose une restriction du champ de liberté de chacun ; d'autre part, le travail productif est fondé sur un rapport de force entre salariés et employeurs, et plus largement entre travail et capital qui implique l'exercice d'un pouvoir qui a pour visée la maîtrise de ce rapport de force, soit la docilité «politique» des salariés. Enfin, comme le rappelle judicieusement D. Linhart (2001), l'efficacité productive n'est pas inscrite dans le contrat de travail : si le salarié met une part de sa liberté et de son temps à la disposition de l'employeur, ce dernier n'est jamais assuré que ce temps sera utilisé de la façon la plus efficiente, ce qui l'amène à envisager des moyens pour s'assurer de cette efficacité ou de cette utilité optimale (dans un contexte donné). C'est ce rapport docilité/utilité que vise le gouvernement des salariés, et ce fut la force des disciplines, comme l'a montré Foucault (1975), que d'articuler ces deux dimensions dans un mouvement d'appui mutuel: agir sur l'individu pour le rendre d'autant plus utile qu'il est docile et inversement. Mais les disciplines ne sont pas seulement des procédures de mises en soumission des corps ; elles ont aussi pour effet la constitution du sujet discipliné.

La communication est progressivement devenue une dimension importante du gouvernement des salariés, un vecteur de cette maîtrise du rapport utilité/docilité, à travers notamment des pratiques de gestion relationnelle (Illouz, 2006) et de gestion symbolique (Floris, 1996 ; Olivesi, 2006). Ceci témoigne aussi d'un déplacement du lieu d'application du pouvoir : c'est moins le corps qui est objet et cible de pouvoir que la psyché (de Gaulejac, 2005; Heller, 2005). Une conséquence est qu'à la différence des disciplines la constitution de soi n'est pas l'effet (possible) d'une technique de gouvernement, mais l'objet même de la technique. C'est le rôle des sémiotechniques que 
Foucault (1975) aborde brièvement; elles sont au centre de notre réflexion. La communication réfère ici à des pratiques de gestion symbolique, mais l'objet même de cette gestion concerne moins le rapport de l'individu au monde que celui de l'individu à lui-même.

\section{La reconnaissance comme opérateur de l'assujettissement}

Notre propos se situant au niveau des généralités, sans ancrage de la communication dans une ou des entreprises particulières, nous proposons d'aborder notre hypothèse de départ à partir de l'évocation de quelques pratiques, situations ou discours qu'il est possible de repérer dans certaines entreprises ${ }^{45}$. Ainsi, quels rapports peut-on établir entre un film qui exhorte les salariés à se mobiliser pour l'entreprise, un entretien d'évaluation du «savoir-être », une séance de coaching visant le développement de l'assertivité, ou l'amélioration des capacités relationnelles, une sensibilisation aux valeurs de l'entreprise dans le cadre de l'accueil de nouveaux embauchés, un article de journal interne qui rappelle la nécessité de « satisfaire » voire «d'enchanter» le client, l'exercice de critiques croisées auquel doit se prêter un manager dans le cadre d'une démarche dite de $360^{\circ}$, les échanges entre un chargé de recrutement et un candidat à l'embauche sur les résultats d'un test de personnalité, une convention sur une île paradisiaque, etc. ?

Tous ces exemples touchent au domaine des comportements au travail et à leur régulation. Un salarié n'est pas forcément pris dans toutes ces pratiques ; certaines concernent les managers, d'autres les salariés qui sont en contact avec les clients, etc. D'une manière générale peu de salariés échappent à l'une ou l'autre de ces pratiques. Mais toutes impliquent l'individu dans la définition d'un rapport à soi et aux autres. Et enfin toutes renvoient à l'idée de reconnaissance.

Il y a deux façons de définir la reconnaissance : dans les termes d'une identification et dans ceux d'une affirmation. La première acception peut être entendue de deux manières différentes : comme un repérage, une évaluation ou une description et comme une appropriation ou une intériorisation (sens psychanalytique). Quant à l'affirmation, elle concerne l'individu dans ses qualités et capacités. Cette acception

45 La notion d'entreprise est ici une facilité de langage. La disparité des réalités auxquelles renvoie cette notion oblige à préciser. Les principales caractéristiques que nous retiendrons sont les suivantes : activité industrielle et commerciale, recherche de profit, division du travail, salariat. 
Dossier : Pour une approche communicationnelle de l'individu au travail

renvoie aux travaux d'A. Honneth $(2006 ; 2007)$, qui ont influencé notre approche.

\section{Reconnaissance-appropriation}

D. Linhart (1994) a mis en évidence que la modernisation des entreprises dans les années 1980 portait moins sur l'organisation du travail que sur le salarié lui-même ; elle relevait d'une demande plus grande d'implication subjective ${ }^{46}$ par le biais de pratiques de participation, visant l'adhésion à l'entreprise à ses objectifs et à ses critères de performance. Cette demande dessine en creux la figure renouvelée du salarié. Celle-ci résulte entre autres choses de la convergence entre une exigence de modifier les conditions de la mise au travail portée par les mouvements sociaux des années 1960 et une exigence d'adapter l'outil de production et la force de travail aux conditions de la guerre économique. Par ailleurs, ce n'est pas seulement un certain rapport au travail dans l'entreprise qui a changé ; l'incitation à être responsable de soi, à s'appuyer sur soi pour inventer sa vie, dont parle, par exemple, Ehrenberg (1995) pour définir la place de l'individu dans la société contemporaine, constitue la toile de fond d'un renouvellement du sens du travail comme vecteur de cette construction et terrain de cette responsabilisation. Etre porteur d'un projet ou désirer s'investir dans un projet devient ainsi, aujourd'hui, le repère qui définit l'individu soucieux de se prendre en charge et de se réaliser. Dès lors, l'entreprise s'envisage comme un support d'une réalisation de soi articulée à la performance économique, par le dépassement ou encore le développement constant de ses capacités. C'est ce qu'expriment, par exemple, certaines annonces d'emplois qui insistent sur le rôle de l'entreprise comme accompagnatrice du projet du candidat, comme terrain d'expression de ses capacités et de ses talents. Ces annonces ne sont pas seulement des promesses: elles définissent comment il faut être par rapport au travail productif. Et dans ce registre de sens, le salarié sera d'autant plus responsable de lui-même qu'il s'impliquera dans/pour l'entreprise et qu'il manifestera ses capacités d'adaptation, de flexibilité ainsi que son souci de maintenir son niveau d'employabilité.

Ce renouvellement du rapport à l'entreprise et au travail, qui induit une figure nouvelle du salarié, s'exprime plus largement dans les médias d'entreprise. Des valeurs, des modèles de conduites y sont

${ }^{46}$ De nombreux travaux en sociologie mettent en évidence que cette demande relève de la contrainte et exprime un renouvellement des formes de domination au travail (voir notamment J.-P. Durand et M.-C. Floch, 2006) 
diffusés et sont relayés par les managers dans le cadre d'échanges institués avec les subordonnés ou par des formateurs dans le cadre de séminaires. Des figures héroïques sont proposées comme modèle d'un éthos du travail productif, comme celle du sportif, qui reflète le mieux l'engagement et la réalisation de soi dans l'action, l'effort et la performance. Mais plus largement, ce sont des attitudes, des modes de raisonnement, des manières d'appréhender le monde, les relations à soi et aux autres (aux clients, aux subordonnés, aux collègues) soustendues par des logiques d'efficacité et de coopération, qui sont proposées à l'identification.

Cependant cette reconnaissance-appropriation ne se limite pas à un rapport du salarié à des contenus normatifs disons exogènes : comme l'a montré Charaudeau (1983), un acte de langage ne se réduit pas à un échange entre un JE sujet-producteur de cet acte et un TU sujetinterlocuteur de cet acte, mais implique les représentations que chacun se fait de l'autre. Autrement dit, dans la production de l'acte (pour ne s'en tenir qu'à cet aspect), JE s'adresse à un TU-destinataire qui est une représentation de TU. Ainsi, dans la manière de s'adresser à l'autre, par l'usage d'un certain lexique, ce sont des caractéristiques identitaires qui sont exprimées, caractéristiques que le TU-interprétant peut accepter ou refuser, auxquelles, donc, il s'identifie plus ou moins. Ici aussi, on est dans le cas d'une figure placée devant soi, mais c'est aussi une représentation de soi, un pari d'identité. Elle peut être également une assignation, consistant à faire comme si le travailleur à qui on s'adresse a les caractéristiques qu'on lui assigne, avec ce que cela implique d'obligations ou d'incitations de conduites. Un exemple est donné par A. Honneth dans La société du mépris, celui de la requalification des salariés en «travailleur-entrepreneur» (Honneth, 2006 : 269) et dont les implications, lorsqu'on s'adresse à eux en ces termes, sont un nouveau rapport au travail tel que nous l'évoquions précédemment, à savoir «que les sujets ne doivent plus comprendre leurs activités comme l'exercice d'une nécessité, mais comme l'épanouissement d'une vocation» (Honneth, 2006: 269). Cette sollicitation du salarié pour l'appropriation de certains modèles de comportements, certaines dispositions ou encore d'une figure particulière de lui-même renvoie la communication à une interpellation, au sens où l'entend Althusser à propos de l'idéologie (1976) dont l'enjeu est la transformation de l'individu-salarié en sujetsalarié (sujet constitué dans/par l'idéologie).

\section{Reconnaissance-description}

Mais il y a une autre manière de l'interpeller, et ce dans un mouvement inverse qui consiste à repérer ce qui le caractérise, à 
Dossier : Pour une approche communicationnelle de l'individu au travail

reconnaître en lui ces modèles et ces normes ainsi que le terrain propice à leur développement.

Au cours des années 1990, un nouveau type d'évaluation a été mis en place, portant sur le "savoir-être », terme qui désigne un ensemble hétérogène comprenant des qualités morales, des dispositions, des capacités relationnelles. Une particularité de cette évaluation est de supposer l'existence d'un lien entre des comportements dans le cadre $\mathrm{du}$ travail (voire les résultats $\mathrm{du}$ travail) et des critères personnologiques (Bellier, 2000). Dans le même temps des pratiques plus anciennes, comme par exemple les tests de personnalité, ont pu s'étendre avec le développement de logiciels d'expertise psychologique. Enfin, à ces pratiques s'ajoutent toutes les techniques de décryptage des comportements, qui ont vocation de mettre au jour les modes de fonctionnement, les blocages internes, les «états du moi » actualisés dans la gestuelle, le langage, etc. (PNL, Analyse Transactionnelle, Process Communication...).

Ces pratiques ne visent pas tous les salariés : le coaching, associé à une démarche d'amélioration de certaines capacités, proche parfois de la démarche thérapeutique, s'adresse plutôt aux cadres; les tests de personnalité ou l'évaluation du «savoir être», en revanche, sont appliqués à un plus large éventail de salariés (et de candidats à l'embauche).

Toutes ces techniques et pratiques ont vocation en premier lieu à décrire, à identifier. Toutefois, leur prétention à décrire n'est jamais qu'une illusion, comme l'a montré J.-L. Beauvois (1994) ; ce qui est repéré ce n'est pas ce que l'on est, mais ce que l'on vaut, c'est-à-dire nos utilités sociales. La pertinence de ces représentations de soi suppose un processus d'identification-appropriation (que l'illusion de la description contribue à favoriser); dans cet esprit, le dialogue avec l'évaluateur peut être entendu comme une contribution visant l'attachement de l'individu à cette représentation de lui-même (Heller, 2006) et la condition préalable à l'acceptation volontaire et motivée d'un travail sur soi dans une perspective d'amélioration. Cet attachement à une figure de soi est aussi attachement à la norme, en raison des orientations positives ou négatives qu'elle véhicule (en tant qu'utilités sociales). Et l'objectivité supposée de la représentation contribue à ce que le travail sur soi puisse s'envisager comme un travail pour soi (et contribue effectivement au développement d'un rapport à soi positif).

À un autre niveau d'analyse, on ne peut négliger le rôle de ces pratiques dans la diffusion d'un vocabulaire permettant de tenir un 
discours sur soi, et aussi sur les autres, de savoirs permettant de se penser à travers des catégories (Illouz, 2006) ; elles contribuent aussi à maintenir chez le salarié une préoccupation du rapport à soi, un soi toujours potentiellement problématique, qui risque à tout instant de trahir.

Enfin, la portée assujettissante de ces pratiques tient également à ceci que cette mise en relation entre conduites et critères personnologiques suppose de considérer que l'efficacité et la force utile du salarié sont logées en lui, et donc qu'il est responsable de ce qui lui arrive (Bellier, 2000). Ces pratiques instituent ainsi une pédagogie libérale remarquable dans la figure du «salarié-entrepreneur », qui renvoie à une conception de l'homme comme capital (Foucault, 2004).

\section{Reconnaissance-affirmation}

Le concept de reconnaissance fait ici référence au travail d'A. Honneth, pour qui il désigne «des pratiques ou des conceptions par lesquelles des sujets individuels ou des groupes sociaux se voient confirmés dans certaines de leurs qualités » (2006 : 252). La pratique de la reconnaissance est considérée comme critère pour penser la normalité d'une société, définie comme une société qui offre à ses membres les conditions de pouvoir se réaliser. C'est en raison du rôle de la reconnaissance dans la constitution de l'identité des sujets et d'un rapport positif à soi, que celle-ci peut effectivement être considérée comme condition de cette réalisation. La reconnaissance est envisagée chez Honneth comme acte moral dont la validité dépend donc de certaines restrictions. En particulier, cet acte doit être «l'expression d'une intention autonome», non liée à «des attitudes positives allant inévitablement de pair avec la poursuite d'autres intérêts de l'interaction » (Honneth, 2006 : 253). Le travail étant une activité qui permet à l'individu de développer et de mettre en oeuvre des capacités, l'entreprise apparaît comme un terrain privilégié pour de tels actes. Mais ils sont loin d'être toujours autonomes, dans la mesure où ils sont aussi une dimension du management (Heller, 2009). C'est d'ailleurs un point abordé par Honneth $(2006)^{47}$ que la reconnaissance puisse relever d'une démarche stratégique, consistant donc à affirmer l'individu dans ses qualités et capacités afin d'en tirer des effets d'utilité et de docilité.

La mise en valeur de salariés à l'occasion de conventions, de remises de prix ou de médailles, d'éloges publics ou dans le cadre d'un

\footnotetext{
${ }^{47}$ En particulier le chapitre 6 intitulé La reconnaissance comme idéologie.
} 
Dossier : Pour une approche communicationnelle de l'individu au travail

entretien d'évaluation, la photo du meilleur vendeur dans le journal interne, etc. témoignent d'expressions particulières de la reconnaissance qui ne se limitent pas aux relations intersubjectives. Elles n'ont pas forcément vocation à entraîner des effets d'utilité/docilité ; mais l'absence de cette vocation ne signifie pas que de tels effets ne se produisent pas chez ceux qui sont visés par la reconnaissance ou chez ceux qui y aspirent.

La soumission aux exigences du pouvoir, quand elle est accompagnée de reconnaissance, peut ainsi être un moyen de maintenir un rapport à soi positif ; elle peut aussi être une condition de validité des qualités qui sont reconnues (autrement dit le fait de ne pas répondre aux exigences du pouvoir remettrait en cause cette qualité). Ce que nous soulignons, de façon sommaire, c'est l'existence d'un rapport entre reconnaissance-affirmation et soumission. Il est ici ramené à quelque ruse du pouvoir étayée sur un désir de reconnaissance. Cette position est réductrice et ne tient pas compte de l'hypothèse, notamment développée par J. Butler (2002), de notre dépendance psychique à l'égard du pouvoir, du rôle de celui-ci comme condition de notre existence sociale, qui permet de comprendre la force de ce rapport.

Si l'on considère maintenant la reconnaissance-affirmation non pas dans son rapport à la soumission mais dans son rapport à la construction de soi, une question vient à l'esprit qui est celle de la définition des critères de cette reconnaissance ${ }^{48}$. Car si celle-ci renvoie effectivement aux qualités et capacités d'un individu ou d'un groupe, elle suppose un cadre qui fait que telle capacité d'un individu ou d'un groupe peut être dotée d'une valeur positive, qui permettra effectivement à cet individu ou à ce groupe d'éprouver un sentiment de valeur, vecteur de l'estime de soi. Reconnaître quelqu'un c'est donc le placer dans un cadre de valeurs ; l'estime de soi que l'on peut tirer de cette reconnaissance dépend de l'appropriation de ce cadre. Inversement, l'appropriation de ce cadre et son actualisation dans des conduites est une condition pour être reconnu.

Si les directions diffusent des modèles de comportements, des valeurs, si elles promeuvent tel ou tel type de qualité, on peut supposer que ces éléments sont aussi ceux sur lesquels vont porter la reconnaissancedescription et la reconnaissance-affirmation. Dès lors, la reconnaissance-affirmation qui est une condition de la construction

\footnotetext{
${ }^{48} \mathrm{Ce}$ qui est une autre manière d'interroger le rapport entre reconnaissance et pouvoir, construction de soi et soumission.
} 
d'un rapport à soi positif peut se comprendre comme une forme de renforcement au sens psychosociologique du terme pour l'appropriation et l'intériorisation des critères de valeur définis par les directions. Autrement dit, cette reconnaissance participe de la construction d'un rapport à soi défini par l'entreprise. Mais aussi, elle institue l'entreprise en soi. C'est le cas plus particulièrement lorsque la reconnaissance-affirmation ne découle pas seulement de l'activité concrète du salarié dans l'entreprise, et de laquelle on en déduit éventuellement des qualités intrinsèques, mais lorsqu'elle porte directement sur les qualités intrinsèques de l'individu. C'est toute l'ambivalence des pratiques psychologisantes que nous avons abordées précédemment : l'identification de telle ou telle disposition, caractéristique, tendance comportementale chez le salarié est aussi évaluation (selon des critères d'utilité/docilité, mais occultés par l'objectivité supposée de l'évaluation) et aussi, par ce biais, un acte de reconnaissance-affirmation qui noue de façon intime le rapport à soi à ces critères...

Ainsi, la communication en entreprise, entendue comme diffusion et circulation de discours normatifs, de figures particulières de salariés, d'actes de description et d'affirmation et qui engagent des processus d'identification, implique à différents niveaux la notion de reconnaissance. L'articulation entre ces niveaux éclaire le mouvement par lequel la communication intervient, dans un rapport de soi à la norme, dans la constitution et l'attachement à soi, la soumission aux autres et comment se fait le lien entre les deux versants de l'assujettissement.

Cette logique assujettissante de la communication peut encore être exprimée de la manière suivante : la communication en entreprise se présente primo comme mise en circulation de modèles, de normes, à intérioriser, deuxio comme pratique de production ou co-production d'une vérité sur soi qui intègre ces normes et tertio comme pratique d'affirmation positive de soi fondée sur celles-ci. Vérité sur soi et affirmation positive contribuent à produire l'attachement à soi tout en évacuant la norme ${ }^{49}$ comme véritable objet d'attachement. Cet attachement favorise la soumission aux autres, d'une part en raison de la norme qui noue le rapport positif à soi à des critères d'utilité et de performance et d'autre part en raison de la reconnaissance-affirmation

\footnotetext{
${ }^{49}$ Norme pourtant contenue dans cette vérité et cette affirmation.
} 
Dossier : Pour une approche communicationnelle de l'individu au travail

produite par le pouvoir et garante de l'estime de soi (et de l'existence sociale $)^{50}$.

\section{Conclusion}

L'hypothèse que nous avons développée ne prend pas en compte la complexité des échanges au sein des entreprises, la diversité des normes qui y sont produites et qui s'y diffusent, la diversité des modes de constructions identitaires et d'attachement à soi qui s'y déploient, la diversité des moyens de soumettre les salariés ou d'en affirmer les qualités hors de pratiques communicationnelles. Notre principal souci était de comprendre les enjeux politiques d'une attention accrue dans de nombreuses entreprises pour l'identité, la singularité, la subjectivité des salariés et qui se manifeste dans la communication. Cet enjeu, exprimé ici en terme de logique d'assujettissement, renvoie aussi à une forme de pouvoir qu'on pourrait appeler gouvernementalité communicationnelle ou encore un dispositif de reconnaissance. La visée critique de notre approche de la communication n'est ni la norme, ni la socialisation, ni même le pouvoir, mais les enjeux de domination qui découlent de la construction (communicationnelle) d'un rapport à soi, ainsi que le sens de cette construction (qui fait de l'individu une ressource ou un capital). L'opérationnalité de cette logique, cependant, est loin d'être effective ; il est probable même que les entreprises soient formées d'une majorité de «mauvais sujets » au sens de cette logique. Cette limite ne signifie pas pour autant qu'elle n'opère pas. D'abord parce qu'il y a aussi des «bons sujets » dans l'entreprise, des sujets qui «marchent tout seul » (pour reprendre une formule d'Althusser, 1976: 121); ensuite parce qu'à défaut de transformer le salarié, le rapport entre normes/soi/affirmation a une portée contraignante qui oblige parfois à jouer le jeu d'un éthos professionnel dans lequel le salarié ne se reconnaît pas, mais qui est une condition pour être intégré et pour être reconnu... (Auger, Balazs, 1996 ; De Gaulejac, 2001).

\section{Bibliographie}

ALTHUSSER L. Idéologie et appareils idéologiques d'Etat. in: ALTHUSSER L. Positions. Paris : Editions Sociales, 1976, pp.67126.

${ }^{50}$ De ce point de vue, les «autres» auxquels on est soumis ne sont pas seulement des pouvoirs incarnés, mais des principes, des objectifs et la logique du système capitaliste dans lequel le salarié est pris. 
BALAZS G. FAGUER J-P., Une nouvelle forme de management : l'évaluation. in Actes de la recherche en sciences sociales, 1996, $\mathrm{N}^{\circ} 114$, pp. 68-78.

BUTLER J. La vie psychique du pouvoir. Paris : Léo Scheer, 2002, $310 \mathrm{p}$.

BEAUVOIS J.-L. Traité de la servitude libérale. Paris : Dunod, 1994, $247 \mathrm{p}$.

BELLIER S. Le savoir-être dans l'entreprise, utilité en gestion des ressources humaines. Paris : Vuibert, 2000, 202 p.

CHARAUDEAU P. Langage et discours. Eléments de sémiolinguistique (théorie et pratique). Paris : Hachette, 1983, $176 \mathrm{p}$.

DURAND J.-P. \& LE FLOCH M.-C. (dir.) La question $d u$ consentement au travail. De la servitude volontaire à l'implication contrainte. Paris : L'Harmattan, 2006, 311 p.

EHRENBERG A., L'individu incertain. Paris: Hachette, Pluriel, 1995, $351 \mathrm{p}$.

FLORIS B. La communication managériale, la modernisation symbolique des entreprises. Grenoble : PUG, 1996, 272 p.

FOUCAULT M. Surveiller et punir, Paris : Gallimard, 1975, 362 p.

FOUCAULT M. Le sujet et le pouvoir. in Dits et Ecrits. t. IV. Paris : Gallimard, 1994a, pp. 222-242.

FOUCAULT M. Les techniques de soi. in Dits et Ecrits. t. IV, Paris : Gallimard, 1994b, pp. 783-812.

FOUCAULT M. Naissance de la biopolitique, Cours au collège de France 1978-1979. Paris : Gallimard, 2004, 355 p.

De GAULEJAC V. L'abstraction et la psychologisation du pouvoir dans l'entreprise hypermoderne. in: Les cahiers du changement, 2001, nº, pp. 25-32.

De GAULEJAC V., La société malade de la gestion, idéologie gestionnaire, pouvoir managérial et harcèlement social. Paris : Seuil, 2005, $280 \mathrm{p}$.

HELLER T. Savoir-être, pouvoir et enjeux identitaires : une critique de l'idéologie du soi. in : Communication et Organisation, 2004, n²4, pp. 73-88.

HELLER T. Performanse: les enjeux d'une confession informatisée. in: Terminal, 2006, n ${ }^{\circ} 95-96$, p. 233-249. 
Dossier : Pour une approche communicationnelle de l'individu au travail

HELLER T. De l'anatomopolitique à la psychopolitique. in : Etudes de Communication, 2005, $\mathrm{n}^{\circ} 28$, pp. 59-76.

HELLER T. Reconnaissance et gouvernement des salariés. Au-delà du mépris. in : Questions de communication, 2009, n 15, pp. 93-107.

HONNETH A. La société du mépris, vers une nouvelle théorie critique. Paris : La Découverte, 2006, 341 p.

HONNETH A. La lutte pour la reconnaissance. Paris : Cerf, 2007, $232 \mathrm{p}$.

ILLOUZ E. Les sentiments du capitalisme. Paris : Seuil, 2006, 204 p.

LINHART D. La modernisation des entreprises. Paris: La Découverte, Repères, 1994, 124 p.

LINHART D. Le rôle de la communication dans le management néoet post-taylorien. in: Terminal, 2001, n ${ }^{\circ} 85$, pp. 157-162.

OLIVESI S. La communication au travail, une critique des nouvelles formes de pouvoir dans les entreprises. $2^{\mathrm{ème}}$ édition. Grenoble : PUG, 2006, 198 p. 